Revue d'histoire de l'Amérique française

REVUE D.HISTOIRE DE L'AMÉRIQUE FRANÇAISE

\title{
La réforme de la justice pénale bas-canadienne : Le cas des assauts communs à Québec
}

\section{Martin Dufresne}

Volume 53, numéro 2, automne 1999

URI : https://id.erudit.org/iderudit/005405ar

DOI : https://doi.org/10.7202/005405ar

Aller au sommaire du numéro

Éditeur(s)

Institut d'histoire de l'Amérique française

ISSN

0035-2357 (imprimé)

1492-1383 (numérique)

Découvrir la revue

Citer cet article

Dufresne, M. (1999). La réforme de la justice pénale bas-canadienne : Le cas des assauts communs à Québec. Revue d'histoire de l'Amérique française, 53(2), 247-275. https://doi.org/10.7202/005405ar
Résumé de l'article

L'auteur décrit à partir d'archives et de textes juridiques la transformation du mode d'administration de la justice urbaine en matière d'assaut commun. Cette transformation est significative, non seulement parce qu'elle met progressivement au rancart la justice des juges de paix, mais aussi parce qu'elle bouscule les rapports entre modes judiciaire et infrajudiciaire de règlement de ces types de conflits. S'inspirant d'une stratégie pénale axée sur la certitude de la punition, le gouvernement met en place des conditions qui facilitent la judiciarisation de ces conflits et qui contribuent à faire de l'assaut commun un crime plutôt qu'une affaire privée. 


\title{
LA RÉFORME \\ DE LA JUSTICE PÉNALE BAS-CANADIENNE LE CAS DES ASSAUTS COMMUNS À QUÉBEC
}

\author{
MARTIN DUFRESNE \\ Département de criminologie \\ Université d'Ottawa
}

\section{RÉSUMÉ}

L'auteur décrit à partir d'archives et de textes juridiques la transformation du mode d'administration de la justice urbaine en matière d'assaut commun. Cette transformation est significative, non seulement parce qu'elle met progressivement au rancart la justice des juges de paix, mais aussi parce qu'elle bouscule les rapports entre modes judiciaire et infrajudiciaire de règlement de ces types de conflits. S'inspirant d'une stratégie pénale axée sur la certitude de la punition, le gouvernement met en place des conditions qui facilitent la judiciarisation de ces conflits et qui contribuent à faire de l'assaut commun un crime plutôt qu'une affaire privée.

\section{ABSTRACT}

Through the use of archives and legal texts, the author describes the transformation of modes of administration of justice in cases of common assault. What makes this transformation significant is not only the gradual exclusion of justices of the peace from urban penal justice, but also a restructuring of the relationship between judiciary and infrajudiciary practices for such conflicts. Inspired by the principle of certainty of punishment, legislative reform facilitated the prosecution of these conflicts. One of the implications of this, was that assaults were increasingly constructed as a public matter rather than a private issue.

$\mathrm{Au}$ cours de la première moitié du XIX ${ }^{\mathrm{e}}$ siècle au Bas-Canada, comme en Angleterre et ailleurs en Occident, le droit pénal et son administration passent par une série de réformes. On assiste notamment à la mise en place d'une nouvelle police, alors qu'en l'espace de quelques années s'estompent la majorité des châtiments publics, marquant la transition vers une stratégie pénale reposant sur la certitude de la punition plutôt que sur l'exemplarité. D'aucuns se mettent à réclamer un pénitencier où l'on réformerait les populations dites oisives et désœuvrées. On cherche en fait dans une justice pénale réformée la garantie d'un ordre social qui puisse accompagner les transformations économiques, politiques et sociales. 
En deçà des préoccupations les plus marquantes comme la police et la peine de mort, le processus judiciaire suscite lui aussi un intérêt croissant, notamment en ce qui concerne les juridictions inférieures. Dès la première session du nouveau gouvernement de 1841, le député Black parvient à faire adopter un ensemble de lois que l'Angleterre avait promulgué plus d'une décennie plus tôt à l'instigation du premier ministre Robert Peel ${ }^{1}$. Le conseil avait tenté de les introduire aussitôt au Bas-Canada, mais ses efforts s'étaient avérés infructueux dans la tourmente qui régnait alors au parlement. Selon Claude Desaulniers, l'adoption de ces lois était d'une grande importance, non seulement pour les nombreux crimes capitaux qu'elles abolissaient, mais aussi parce qu'il s'agissait ni plus ni moins d'une «semi-codification $^{2} »$. En effet, cet effort de consolidation du droit pénal est une pièce importante de la dissolution de la justice pénale d'Ancien Régime. Ces lois prévoient une justice de procédure sommaire pour certaines catégories d'infraction. Qui plus est, au détriment de la cour des sessions trimestrielles de la paix et des juges de paix de la ville, cette justice sommaire sera administrée dans le cadre de la cour de police, avec pour maître d'œuvre un magistrat salarié: l'inspecteur et surintendant de police.

David Philips a décrit quelques aspects de la mise en place de cette vaste réforme du judiciaire en Angleterre ${ }^{3}$. Il a insisté sur l'impuissance grandissante du mode traditionnel d'administration de la justice face à l'urbanisation et aux populations flottantes, en particulier à Londres. D'autres ont insisté davantage sur une augmentation du crime comme moteur du changement. C'est le cas de John C. Weaver qui, dans son travail sur Hamilton, situe les réformes dans la perspective d'une réaction utilitariste aux désordres grandissants ${ }^{4}$.

1. Il s'agit de $4 \& 5$ Vict.(1841), c.24: Acte pour améliorer l'administration de la justice en matière criminelle dans cette province; c.25: Acte pour consolider et amender les lois de cette province qui ont rapport au larcin et autres offenses y relatives; c. 26: Acte pour consolider les statuts de cette province, relatifs aux dommages malicieux causés à la propriété; c. 27: Acte pour consolider et amender les statuts de cette province, relatifs aux offenses contre la personne. Voir Edmond Lareau, Histoire du droit canadien (Montréal, Librairie générale de droit et de jurisprudence, 1888), 302.

2. Claude Desaulniers, «La peine de mort dans la législation criminelle de 1760 à $1892 »$, Revue générale de droit, 8,2 (1977): 152, 167-169.

3. David Philips, «A New Engine of Power and Authority: The Institutionalization of Law-Enforcement in England 1780-1830», dans V. A. C. Gatrell et. al., dir., Crime and the Law. The Social History of Crime in Western Europe Since 1500 (London, Europa Publications, 1980), 155-189.

4. John C. Weaver, Crimes, Constables, and the Courts. Order and Transgression in a Canadian City, 1816-1970 (Montréal/Kingston, McGill-Queen's University Press, 1995). D'autres se sont penchés sur la mise en place d'une cour de police et de corps de police, en 
Dans son ouvrage sur la ville de Québec, bien qu'il ne fasse qu'effleurer le judiciaire, David Thierry Ruddel prétend que certaines réformes suivent le rythme d'une augmentation du crime, augmentation qui s'expliquerait par les changements de l'économie et surtout par l'afflux d'immigrants 5 .

Certes, la ville de Québec, en pleine expansion depuis le début du siècle, se trouve au carrefour d'une économie du bois qui va croissante et à laquelle se greffent des flux migratoires. Cependant, cette prétendue augmentation du crime doit être soumise à une analyse critique. Si l'historien trouve une augmentation de dossiers à quelque étape que ce soit dans le processus judiciaire, celle-ci peut bien s'expliquer par des changements ponctuels dans l'administration de la justice pénale, plutôt que par une augmentation réelle du crime. Resserrer les mailles du processus judiciaire, adopter des mesures qui facilitent la judiciarisation et mener une politique répressive soutenue à l'égard de certains types d'infractions peuvent bien expliquer les fluctuations, si abruptes soient-elles, du volume de ce qu'on appelle le crime. De la même manière, accepter pour preuve l'opinion de certains observateurs de l'époque selon laquelle le crime augmenterait consiste à leur accorder une confiance fort discutable. Dénoncer une augmentation du crime peut bien faire partie d'une stratégie de réforme, visant à provoquer un réaménagement de la justice pénale.

Allan Greer nous rappelle que si les nouveaux corps de police remplissent un rôle d'ordre et de sécurité dans les rues, ils font aussi partie de cette vaste entreprise de réforme sociale qui cherche à façonner les conduites et à forger un nouvel ordre social, signe d'une tendance de l'État à empiéter sur la société civile ${ }^{6}$. Avant la nouvelle police, la réforme des masses devient un objet de préoccupation, notamment par des discours rapprochant pauvreté et crime, puis par un recours croissant à la prison pour les populations dites oisives et vicieuses ${ }^{7}$. Le droit pénal et son administration n'échappent pas aux critiques, aux projets et aux

insistant toutefois moins sur le droit et le judiciaire que sur la problématique des classes sociales, sur celle de la légitimité ou encore sur le rôle de «service» de la police. Voir la synthèse de Greg Marquis, «Towards a Canadian Police Historiography», dans Louis A. Knafla et Susan W. S. Binnie, dir., Law, Society, and the State (Toronto, University of Toronto Press, 1984).

5. David Thierry Ruddel, Québec, 1765-1832 (Hull, Musée canadien des civilisations, 1991), 184-185.

6. Allan Greer, «The Birth of Police in Canada », dans Allan Greer et Ian Radforth, dir., Colonial Leviathan. State Formation in Mid-Nineteehth-Century Canada (Toronto, University of Toronto Press, 1992), 17-49.

7. Jean-Marie Fecteau, Un nouvel ordre des choses: la pauvreté, le crime, l'État au Québec, de la fin du XVIII e siècle à 1840 (Montréal, VLB éditeur, 1989), 173-206; Martin Dufresne, La justice pénale et la définition du crime à Québec, 1830-1860, thèse de doctorat (histoire), Université d'Ottawa, 1997. 
velléités de réforme. En témoigne la nomination par le gouvernement d'un président de la cour des sessions trimestrielles de la paix en 1810, année mouvementée sur le plan politique ${ }^{8}$.

De ce point de vue, le processus judiciaire se trouve lui aussi soumis au réaménagement institutionnel qui accompagnera la transition au capitalisme industriel ${ }^{9}$. L'appareillage institutionnel, qui constitue une nouvelle figure de l'État au XIX ${ }^{\mathrm{e}}$ siècle, peut compter, entre autres choses, sur une nouvelle police dès 1837 à Québec et bientôt dans les autres villes coloniales, puis sur des administrations municipales et des officiers salariés plutôt que rémunérés selon des honoraires. La transformation du processus judiciaire, qu'implique l'introduction des lois de Peel au Bas-Canada, est significative en ce qu'elle témoigne d'un projet de droit pénal qui s'inspire de l'idée de certitude. Bien qu'elle concerne essentiellement des infractions mineures, elle accompagne les restructurations du corps de police, puis la nomination d'un inspecteur et surintendant de police (magistrat de police). Ensuite, elle participe d'une transformation du rapport entre les citoyens et la justice pénale, quant au rôle des citoyens et quant à une criminalisation des infractions d'assauts et éventuellement des petits vols ${ }^{10}$. Cette transformation modifie du même coup l'articulation des modes judiciaires et infrajudiciaires de règlement des conflits par la mise en place de moyens visant à faciliter la judiciarisation et à resserrer le processus judiciaire.

C'est ce que nous allons voir en nous penchant sur les parcours qu'empruntent les cas d'assaut dans le processus judiciaire avant et après l'adoption des lois de Peel. Nous ferons appel à certaines des archives conservées au greffe de la paix de Québec ${ }^{11}$. Les dossiers

8. Sur la question, on ne peut contourner l'ouvrage de Donald Fyson pour Montréal, Criminal Justice, Civil Society and the Local State: The Justices of the Peace in the District of Montreal, 1764-1830, thèse de doctorat (histoire), Université de Montréal, 1995, première partie, chapitre IV: "The Duties and Powers of the Justices». J.-M. Fecteau a aussi noté des efforts entrepris afin de donner davantage de poigne à l'appareil de justice pénale. Voir, J.-M. Fecteau, op. cit., 105-107.

9. John A. Dickinson et Brian Young, «Periodization in Quebec History: A Reevaluation», Québec Studies, 12 (1991): 1-10.

10. Voir Allen Steinberg, «Criminal Prosecution, Assault and the Decriminalization of Everyday Life in Nineteenth-Century Philadelphia», International Association for the History of Crime and Criminal Justice, 17 (1992-1993): 66-80.

11. À moins d'une indication contraire, les documents d'archives auxquels nous allons référer - dossiers, actes d'accusation, lettres, représentations du grand jury, calendriers de la prison - proviennent des Archives nationales du Québec à Québec, dans le fonds TP12, S1. Il sera aussi question du registre de la cour des sessions de la paix dans le même fonds, dont nous possédons les volumes pour les années 1823 à 1848 et 1859 à 1878 . Alors que nous entreprenions nos recherches, ces documents subissaient un reclassement. Nous allons donc y référer en indiquant seulement les renseignements relatifs au dossier ou au registre. 
criminels décrivent peu l'événement qui déclenche la poursuite pénale. Néanmoins, ils nous permettent de voir comment s'administre la justice tout au long du processus judiciaire, soit les avenues qu'empruntent les dossiers, la mise en forme pénale d'une querelle ou d'un litige et, enfin, le dénouement de certaines de ces poursuites pénales. Même si à l'époque, la juridiction de la cour des sessions de la paix de Québec couvre un vaste territoire, la majorité des affaires d'assaut qui y sont entendues proviennent de la ville ${ }^{\mathrm{i} 2}$. Précisons qu'il n'est pas question d'estimer un nombre réel d'assauts. Les archives judiciaires se prêtent mal à un tel exercice, non seulement parce que la justice de l'époque ignore de nombreux cas qui ne seront jamais portés à son attention (c'est le chiffre noir de la criminalité), mais aussi parce que les aléas du judiciaire, dont il sera question dans les pages qui suivent, montrent à quel point il serait imprudent de spéculer sur la quantité de crimes. Nous ne chercherons donc pas à évaluer l'impact de la réforme sur le nombre des assauts réellement commis.

\section{LA JUSTICE PÉNALE ET L'INFRAJUDICIAIRE}

À Québec, durant le premier tiers du XIX ${ }^{\mathrm{e}}$ siècle, une cour du banc du Roi agit comme haute cour criminelle (et civile) et siège deux fois l'an. Dans les juridictions inférieures, ce sont des juges de paix qui président aux procès en matière pénale, parfois seuls, à deux ou à plusieurs. Trois d'entre eux, au minimum, s'assemblent quatre fois l'an pour tenir les sessions trimestrielles de la paix qui durent dix jours où, en plus des procès, la cour se penche sur des affaires urbaines comme l'octroi des licences d'auberge, la location des étals des marchés, l'homologation des procès-verbaux de l'inspecteur des chemins, etc. La ville étant sans charte municipale, ce sont des juges de paix qui gouvernent Québec en sessions spéciales et lors des sessions trimestrielles de la paix ${ }^{13}$. La quarantaine de poursuites pénales, qui aboutissent devant cette juridiction lors d'une des sessions, se greffe à d'autres dossiers relatifs à l'administration urbaine. Chaque session de la paix réunit alors une centaine de citoyens appelés à remplir la charge de juré, près d'une autre centaine, ceux-là constables nommés annuellement, et, enfin, des avocats, des individus qui s'intéressent aux affaires urbaines et des curieux.

12. 3 Geo. IV (1823), c.17.

13. De avril 1833 à mai 1836 , la cité fut administrée en corporation. La charte n'est pas renouvelée en 1836; elle ne le sera qu'en 1840, au lendemain des troubles. Voir 1 Guil. IV (1831), c.52 et 4 Vict.(1840), c.35. 
Compte tenu de la concentration de la justice royale dans les villes, compte tenu aussi d'un nombre relativement modeste de dossiers qui aboutissent devant la cour du banc du Roi et devant les sessions trimestrielles de la paix, puis des moyens restreints pour administrer la justice, comme la police, Jean-Marie Fecteau en arrivait à la conclusion qu'à l'époque, la justice royale remplissait une «fonction d'arbitrage suprême de tensions que l'organisation communautaire ne peut résoudre ${ }^{14}{ }$. C'est aussi ce qu'a laissé entendre Allan Greer dans les quelques pages qu'il a consacrées à la question ${ }^{15}$. Nombre de querelles à propos de biens et nombre de menaces ou bagarres se résolvaient par divers mécanismes propres à la communauté, des mécanismes infrajudiciaires, en particulier dans les campagnes. En s'opposant à cette thèse, Donald Fyson s'est efforcé de montrer que, dès le début de ce siècle, non seulement il existe une sorte de corps policier à Montréal, un corps relativement permanent et actif et ce, jusque dans les campagnes qui environnent la ville, mais aussi que les particuliers n'ont pas tous les scrupules que l'on croyait à engager des poursuites judiciaires, notamment pour des affaires mineures ${ }^{16}$. Il existe aussi bon nombre d'officiers qui voient à faire respecter divers règlements et qui participent plus ou moins activement au processus judiciaire, le plus souvent en ce qui concerne les mandats et sommations.

D'une certaine manière, ces thèses ne s'opposent qu'en apparence. S'il faut admettre que la justice pénale peut s'étendre dans les campagnes, il ne faut pas pour autant conclure qu'une participation policière ou celle d'autres officiers de justice implique nécessairement qu'un dossier aboutira à un procès, comme s'il tombait entièrement dans les mains des officiers de justice. En fait, il est tout indiqué de nuancer ce que l'on considère souvent comme une opposition entre judiciaire et infrajudiciaire. Quelques remarques sur la police et sur l'infrajudiciaire s'imposent.

À propos de la police d'abord, nous devons distinguer, parmi ses activités, celles où elle agit de son propre chef, de celles où elle est davantage à la remorque des particuliers. L'existence d'un corps de police relativement permanent n'implique pas nécessairement qu'il

14. J.-M. Fecteau, op. cit., 125. On trouvera une perspective similaire chez Alfred Soman, «Justice et infrajustice en France (XVI $-\mathrm{XVIII}^{\mathrm{e}}$ siècles)», dans Benoît Garnot, dir., L'infrajudiciaire du Moyen Âge à l'époque contemporaine (Dijon, Éditions universitaires de Dijon, 1996), 77-85.

15. Allan Greer, The Patriots and the People. The Rebellion of 1837 in Rural Lower Canada (Toronto, University of Toronto Press, 1993), 91-100.

16. D. Fyson, op. cit. 
exerce une mainmise, voire un monopole sur les poursuites judiciaires. À Québec, avant les rébellions, la police agit de son propre chef essentiellement pour faire la chasse aux matelots déserteurs, aux indigents et aux prostituées qui peuvent être emprisonnées sommairement. C'est aussi elle qui poursuit les vendeurs d'alcool sans permis ${ }^{17}$. Ce qui n'empêche pas les policiers de délivrer des mandats, des sommations et même de mener des enquêtes très élaborées pour certaines affaires, surtout les plus sérieuses.

En ce qui concerne les cas d'assaut commun, le rôle de la police est limité. Il faut non seulement que les particuliers décident de poursuivre, mais ils doivent aussi porter le fardeau d'organiser la poursuite, ce qui implique notamment des frais de justice. Bien que dans la métropole, la plupart des types d'affaires pénales conservent ce caractère privé, il en va différemment dans la colonie où il est fréquent que des officiers de justice se substituent à la partie plaignante devant les $\operatorname{cours}^{18}$. Il n'en demeure pas moins qu'il semble assez difficile pour l'instant de parler d'un monopole de la poursuite pour toutes les affaires pénales, c'est-à-dire d'un monopole où le plaignant est mis à l'écart d'une poursuite entièrement menée par les officiers de justice ${ }^{19}$. Quoi qu'il en soit, en matière d'assaut commun, la police agit essentiellement pour mettre à exécution des ordres de cour, de sorte qu'une affaire de la sorte continue d'être définie et traitée comme une affaire privée. Les particuliers jouissent donc d'une marge de manœuvre dans l'exercice de la justice pénale. Il est toléré qu'ils se retirent de leur propre poursuite durant le processus judiciaire, ce qui met fin à la poursuite.

$\mathrm{Si}$, en règle générale, la police ne joue qu'un rôle accessoire en matière d'assaut commun, qu'en est-il des mécanismes infrajudiciaires? Les travaux récents sur l'infrajudiciaire portent à croire que la justice pénale n'est pas nécessairement le lieu privilégié du

17. Une telle poursuite consiste en une action pénale. Lorsqu'il y a condamnation, la partie plaignante (la police) et le Roi se partagent le montant de l'amende à part égale. Selon toute vraisemblance, ces amendes permettent de défrayer les coûts de la police.

18. En cour du banc du Roi, ce sont les procureur général et solliciteur général qui poursuivent plutôt que les particuliers ayant déposé la plainte. Â la cour des sessions trimestrielles de la paix, le greffier prend parfois ce rôle.

19. Sur la monopolisation de la poursuite, voir Douglas Hay et Francis Snider, «Using the Criminal Law, 1750-1850: Policing, Private Prosecution, and the State», dans D. Hay and F. Snider, dir., Policing and Prosecution in Britain 1750-1850 (Oxford, Clarendon Press, 1989), 3-52; Clive Emsley et Robert D. Storch, «Prosecution and the Police in England Since 1700», International Association for the History of Crime and Criminal Justice Bulletin, 18 (printemps 1993) : 45-57. 
règlement d'affaires qui peuvent néanmoins aboutir devant les tribunaux, entendons d'affaires qui répondent à des critères permettant de les traduire en affaires de justice pénale. On a ainsi rapporté toute une gamme de pratiques en parallèle à la justice pénale. Parfois très formalisées, parfois très informelles, ces pratiques vont de l'acte notarié qui scelle un accord entre les parties pour une réparation quelconque, à la personne qui, en privé, en force une autre à réparer son tort par la menace d'une poursuite parfois lourde de conséquences.

Depuis les travaux de Nicole Castan et d'Alfred Soman ${ }^{20}$, on a insisté sur les rapports entre l'infrajudiciaire, la justice locale et la justice pénale royale, sur le caractère plus ou moins formel des processus à l'œuvre, souvent sur la force d'expansion de la justice royale, auquel cas l'infrajudiciaire se résorberait. Ces rapports peuvent bien s'interpréter sur l'axe de l'autonomie des communautés rurales ou urbaines vis-à-vis du pouvoir de l'État, comme le suggère Benoît Garnot $^{21}$. Ou encore, selon Andrea Zorzi, il faut peut-être nuancer la thèse de l'affirmation progressive de l'État, car il est possible que des transformations des pratiques infrajudiciaires influencent la place qu'occupe la justice officielle 22 . À cet enchevêtrement complexe de modes de régulation informel, local, urbain ou étatique, s'ajoute une autre composante, celle des intentions et stratégies du plaignant qui peut avant tout chercher un dédommagement ou une réparation symbolique lorsqu'il fait appel à la justice ${ }^{23}$.

Ce dernier aspect, relatif au plaignant, s'avère déterminant dans les types d'affaires définies comme privées. De ce point de vue, un recours en justice ou hors justice peut dépendre de la stratégie, laquelle est faite d'intérêts et de perspectives de réparation, de sorte que la stratégie influence le choix d'un mode de règlement. S'offrent

20. Nicole Castan, Justice et répression en Languedoc à l'époque des Lumières (Paris, Flammarion, 1980); Alfred Soman, «Deviance and Criminal Justice in Western Europe, 13001800: An Essay in Structure », Criminal Justice History, 1 (1980) : 3-28; Alfred Soman, «L'infra-justice à Paris d'après les archives notariales », Histoire, économie, société, 3 (1982): 369376; J. H. Baker, «Criminal Courts and Procedure at Common Law 1550-1800», dans J. S. Cockburn, dir., Crime in England 1550-1800 (New Jersey, Princeton, 1977), 16-17.

21. Benoît Garnot, «L'ampleur et les limites de l'infrajudiciaire dans la France d'Ancien Régime $\left(\mathrm{XVI}^{\mathrm{e}}-\mathrm{XVII} \mathrm{e}^{\mathrm{e}}-\mathrm{XVIII}{ }^{\mathrm{e}}\right.$ siècles)», B. Garnot, dir., op. cit., 69-76.

22. Andrea Zorzi, «Conflits et pratiques infrajudiciaires dans les formations politiques italiennes du XIII ${ }^{\mathrm{e}}$ au XV $\mathrm{XV}^{\mathrm{e}}$ siècle», dans B. Garnot, dir., op. cit., 19-36. Sur l'affermissement de l'État centralisateur, voir dans le même ouvrage, Xavier Rousseau, «Entre accommodement local et contrôle étatique. Pratiques judiciaires et non judiciaires dans le règlement des conflits en Europe médiévale et moderne», 87-107.

23. A. Soman, «Deviance and Criminal Justice...», loc. cit.; voir aussi les discussions à ce propos dans B. Garnot, dir., op. cit., 129-139. 
à cet acteur des possibilités, qui dépendent sans doute de rapports de force, mais aussi de partages entre judiciaire et infrajudiciaire, puis de leurs rapports. On peut observer ces partages et parfois une concurrence entre les justices urbaine, locale et plus informelles. Quant à ces rapports, ils s'organisent en fonction au moins d'une donnée: il s'agit de conflits susceptibles de mise en forme pénale. En d'autres mots, la justice pénale peut se trouver suspendue lorsque des pratiques infrajudiciaires sont suivies, mais il peut aussi suffire de peu pour qu'elle s'active. Et, de la même manière, elle a beau s'activer, cela ne veut pas nécessairement dire que l'affaire suivra un cours inéluctable et se dirigera tout droit vers le procès. C'est en partie ce qui permet d'expliquer l'écart entre le volume des démarches de justice entreprises et le volume de ce qui aboutit finalement devant le tribunal et au procès.

En observant les archives judiciaires à Québec, on s'étonne du grand nombre de cas d'assauts trouvés dans des dépositions, comparativement à la faible proportion de ces dossiers qui vont réellement aboutir à un procès. C'est dire que la mécanique infrajudiciaire ne fonctionne pas uniquement en dehors de l'administration de la justice pénale, celle-ci en est plutôt imprégnée. Comme le disait Robert B. Shoemaker, en matière de méfaits au moins («misdemeanour»), la justice pénale s'administre avec flexibilité ${ }^{24}$.

L'évolution de l'administration de la justice en matière d'assaut à Québec relève de ces enjeux. Ainsi, la réforme du judiciaire est à replacer dans un nouveau partage de la justice pénale entre les gouvernements central et local, ce qui va s'opérer par le truchement de la cour de police. Ensuite, la même réforme s'inscrit dans une nouvelle recherche d'efficacité, reposant sur des moyens qui s'accordent mal avec le mode d'administration de la justice d'alors, notamment là où il fait place aux plaignants pour déterminer le cours que va suivre un dossier.

\section{LES ASSAUTS ET LA JUSTICE PÉNALE AVANT LA RÉFORME}

Afin de distinguer les avenues judiciaires que peuvent emprunter les poursuites pénales à Québec durant les années 1830, on peut s'en remettre à trois caractéristiques qui définissent l'administration de la justice en droit pénal anglais ${ }^{25}$. Avec des balises plus ou moins rigides, la loi indique des catégories d'infractions pénales, puis elle prévoit les

24. Robert B. Shoemaker, Prosecution and Punishment. Petty Crime and the Law in London and Rural Middlesex, c. 1660-1725 (Cambridge, Cambridge University Press, 1991).

25. Voir S. F. C. Milsom, Historical Foundations of the Common Law (London, Butterworths, 1969), 353-356. 
tribunaux qui peuvent accueillir les poursuites pour telle ou telle autre infraction (juridictions). La troisième caractéristique, moins explicite du point de vue de la loi, concerne le statut de la personne qui prend l'initiative de la poursuite. Il peut s'agir d'un particulier ou de divers officiers de justice comme le grand connétable, le greffier de la paix ou encore les procureur et solliciteur général. Ce serait refuser toute la complexité du processus judiciaire de considérer que les cours ont pour seule fonction de trancher sur la responsabilité de l'accusé. En fait, les juridictions suivent leur propre procédure et les personnes dont nous venons de faire état remplissent parfois des rôles différents selon l'infraction et selon la juridiction. Enfin, la qualification juridique d'une infraction ne s'impose pas de manière automatique à un événement susceptible d'une judiciarisation.

Cette dernière remarque s'applique tout particulièrement aux affaires d'assaut, où la frontière est souvent mince entre une menace, un assaut, un assaut et batterie ou un assaut avec intention de meurtre. On peut, comme le suggère Jacques Crémazie à l'époque, distinguer des assauts «communs» et des assauts «graves ${ }^{26}$ ». L'assaut commun réfère essentiellement à l'assaut et batterie qui consiste en l'accomplissement d'une violence physique, si minime soit-elle. Il y a assaut et non batterie lorsqu'une personne donne à une autre l'impression qu'elle va la battre, mais qu'elle ne passe pas aux actes. En pratique cependant, des dépositions pour assaut et batterie servent dans une grande diversité de conflits, allant du harcèlement à des querelles relatives à la propriétée ${ }^{7}$.

Les assauts peuvent emprunter deux avenues judiciaires qui ont des conséquences différentes sur plusieurs plans. Le plus souvent dans le cas de menaces, mais aussi fréquemment lorsque des coups sont portés, un plaignant dépose devant un juge de paix dans le but de dénoncer les gestes de l'accusé et de se protéger contre une nouvelle agression. Dans ce type de démarche, que l'on qualifie de "poursuite pour la paix», un plaignant peut obtenir d'un juge que l'accusé soit forcé de s'engager par cautionnement à garder la paix pendant six mois ou un an. On fixe généralement à vingt livres le montant du cautionnement ${ }^{28}$. L'accusé qui se voit imposer cette décision par un juge de paix seul, c'est-à-dire par une procédure sommaire, doit trouver deux personnes qui se portent garantes de sa conduite, en foi de

26. Les lois criminelles anglaises, traduites et compilées de Blackstone, Chitty, Russell et autres criminalistes anglais... (Québec, Fréchette, 1842), 88.

27. A. Steinberg, loc. cit., 66-67; R. B. Shoemaker, op. cit., 53.

28. Le cautionnement pour la paix ou pour bonne conduite semble avoir été utilisé pour toutes sortes d'infractions. Dans le cas de l'Angleterre, voir R. B. Shoemaker, op. cit., 98-101. 
quoi elles s'engagent aussi devant le Roi pour la moitié de la somme imposée au principal intéressé.

À Québec, ils sont des centaines chaque année à dénoncer ainsi un agresseur, soit une femme contre un époux violent, un père contre son fils, un autre contre un voisin, une connaissance ou un étranger. Sans trop de formalité semble-t-il, les juges de paix acquiescent à l'initiative du plaignant; il arrive aussi qu'ils forcent les deux parties à s'engager ainsi à garder la paix. Par exemple, le 15 septembre 1836, Thomas Scott, charretier, dépose «pour la paix» contre un tonnelier du nom de Thomas Canty. Selon les formules d'usage trouvées dans la plupart des dépositions pour assaut et batterie, celle de Scott indique qu'il fut «violently assaulted struck and beat». Chargé de l'affaire, le juge de paix Symes impose à Canty un cautionnement pour la paix. Toutefois, le même jour, Canty dépose une accusation semblable contre Scott, et celui-ci devra aussi s'engager à garder la paix au moment de sa déposition ${ }^{29}$. Il est difficile d'estimer l'effet d'une telle mesure sur la conduite des accusés ${ }^{30}$. Par ailleurs, en décrétant un blâme, la mesure comporte un aspect infamant qui participe au caractère pénal de l'initiative. Nous reviendrons plus loin sur la propriété pénale des poursuites, après avoir examiné l'autre avenue judiciaire qu'empruntent les affaires d'assaut.

Outre cette procédure sommaire de dénonciation, les plaignants peuvent mener leur dossier devant la cour des sessions trimestrielles de la paix, comme dans les cas de petits vols. Ce sont à nouveau des juges, qui font partie de la commission de la paix, qui se pencheront sur l'affaire, mais dans un cadre fort différent. Un dossier d'assaut soumis à cette juridiction suit un parcours complexe, nécessitant d'abord qu'en vertu de la déposition originale, le greffier prépare un document qui constitue l'acte d'accusation («bill of indictment»). Ce document sera soumis à un grand jury lors de l'ouverture de la session, afin de déterminer si la partie plaignante dispose d'une preuve suffisamment étoffée pour que l'affaire passe au procès, auquel cas un petit jury rendra le verdict. Bien qu'une mise en accusation de la sorte s'effectue au nom du Roi, les assauts communs, c'est-à-dire lorsqu'il n'y a pas «intention de commettre» un meurtre ou un viol par

29. T. Scott vs T. Canty, for the peace, 1836-09-15; T. Canty vs T. Scott, for the Quarter session, 1836-09-15. Les cautionnements pour la paix accompagnent les dépositions. Bien qu'il soit indiqué sur la déposition de Canty que le dossier se dirige vers la cour des sessions de la paix, il ne s'y retrouvera pas lors de la prochaine session.

30. Dans les archives consultées au greffe de la paix de Québec, nous n'avons pas trouvé trace de tels cautionnements qui auraient éventuellement été prélevés. Il est néanmoins possible qu'ils l'aient été. 
exemple, conservent leur caractère privé, de sorte qu'il revient au plaignant de débourser les frais de justice ${ }^{31}$. Et dans l'éventualité d'un verdict de culpabilité, la cour choisit une sentence, soit l'amende, soit l'emprisonnement.

En Angleterre, la cour laisse parfois aux parties impliquées dans des causes de la sorte l'occasion de convenir entre elles d'un règlement quelconque. William Blackstone dénonce cette pratique, notamment quand elle a lieu en cour des sessions de la paix où, après un verdict de culpabilité, il arrive que les parties s'entendent et que la cour réduise l'amende en conséquence. Cet usage permet de dédommager le plaignant pour ses frais et pour le tort subi, ce qui évite une poursuite civile. Blackstone s'y oppose, sous prétexte que "prosecutions for assault are by this means too frequently commenced, rather for private lucre than for the great ends of public justice ${ }^{32} »$. Cependant, la pratique ne semble pas avoir été suivie en cour des sessions de la paix à Québec, du moins pas systématiquement selon le registre et selon ce que rapportent les journaux. Nous en reparlerons plus loin.

Si les juges n'ont pas de scrupules à imposer des cautionnements dans les cas de "poursuites pour la paix », par contre les poursuites destinées à la cour des sessions trimestrielles n'aboutissent que rarement à un verdict de culpabilité. En fait, la majorité d'entre elles n'arrivent même pas devant la cour. Loin de rechercher nécessairement une sentence, les plaignants qui ont recours à la justice des tribunaux peuvent bien se satisfaire d'une punition qui se passe d'un verdict de culpabilité ou encore d'accommodements hors cour favorisés par la déposition d'une plainte et tolérés par la justice.

Le nombre de poursuites pour assaut qui disparaissent du processus judiciaire avant la réforme de 1841 est remarquable. Par exemple, nous avons retracé dans les boîtes du greffe de la paix de Québec 138 dossiers indiquant un assaut ou une menace pour les mois d'août, septembre et octobre $1836^{33}$. Les plus importantes catégories sont

31. Ce que Joseph Chitty qualifie de «injury of a private nature». Voir Joseph A. Chitty, A Practical Treatise on the Criminal Law... (Philadelphia, Isaac Riley, édition de R. Peters, 1819), 2.

32. William Blackstone, Commentaries on the Laws of England, vol.4, (London, Thomas Tegg, $7^{\text {th }}$ edition, 1765, 1830), 363-364; Thomas Noon Talfourd, Dickinson's Guide to The Quarter Sessions, and Other Sessions of the Peace (London, S. Sweet, V. \& R. Stevens..., 1845, $6^{\text {th }}$ edition), 456.

33. Nous avons choisi 1836 au hasard. Un survol du registre n'indique rien qui semble différer depuis les années 1820. Le trimestre d'automne est néanmoins particulier en ce qu'il est habituellement celui qui compte le plus de dossiers. Mais d'année en année, la proportion de dossiers dans chacun des trimestres reste à peu près la même. Cent quatre-vingt-un dossiers 
celles de l'assaut et batterie (80 cas), de menace ( 24 cas), puis d'assaut et menace (9 cas). Ce qui nous importe, c'est que sur 78 de ces 138 dossiers, il est indiqué que la déposition vise un procès devant la cour des sessions trimestrielles de la paix qui se tiendra à la toute fin d'octobre; tandis que les 60 autres consistent en «déposition pour la paix » et elles mènent effectivement à un cautionnement. Or, bien que l'on compte 78 dossiers d'assauts destinés à un procès, seulement 25 d'entre eux sont réellement déposés à l'ouverture de la session, le 21 octobre, puis soumis au grand jury. Et seulement huit de ces derniers portent sur des assauts et batterie, les autres concernant des assauts contre un officier ou des assauts avec intention de meurtre. D'après les notes inscrites sur les dépositions, on peut observer que deux dossiers destinés aux sessions de la paix sont réglés par une entente entre les parties, probablement avec l'assentiment du juge de paix. Par ailleurs, on ne sait rien des 53 autres dépositions, dont 42 concernent un assaut et batterie. Le 15 septembre 1836, W. Kendall dépose pour un procès à la prochaine session de la paix, expliquant que son père et lui ont été insultés et frappés par H. Jones. Or, Jones dépose lui aussi contre les Kendall sous le même prétexte, se plaçant ainsi sur le même pied que ses adversaires. On ne sait rien de plus de la suite de l'affaire, sinon que ni l'une ni l'autre de ces deux poursuites n'apparaissent dans le registre lors de la session d'octobre ${ }^{34}$.

On ne saurait dire ce qu'il advient de ces affaires entre particuliers, bien que l'on puisse croire que certaines d'entre elles se soient réglées par un accommodement, pratique répandue à l'époque. On se sert alors de la menace d'une poursuite pour forcer un adversaire à accepter de négocier une réparation. C'est d'ailleurs ce que fera remarquer le capitaine Wetherall, alors magistrat de police, à l'assistant secrétaire civil William Coffin - futur commissaire de police pour tout le Bas-Canada - en lui expliquant qu'au nord de Montréal,

\footnotetext{
furent retracés au greffe de la paix de Québec pour cette période de trois mois. Il est probable que ce nombre soit au-dessous du nombre réel de dossiers. Peu nous importe, étant donné que le registre nous indique le nombre de dossiers qui aboutissent éventuellement en cour et que, par ailleurs, nous ne cherchons pas à mesurer ou à estimer un nombre réel d'assauts. Si des dossiers nous échappent, cela ne ferait qu'augmenter cet écart entre le nombre de dépositions et le nombre de procès. Les dossiers d'assaut sont qualifiés de diverses façons. Nous avons retenu ceux qui portent l'inscription suivante: assault and battery; assault and threats; threats \& $c$.; assault \& c. ; assault with intent to murder; assault with intent to ravish; assault on a constablemagistrate-pilot; assault with a knife; assault \& breach of the peace; assault \& breaking windows. Nous n'avons pas retenu les huit dossiers dans lesquels il est question de riot, puisqu'ils impliquent plusieurs personnes.

34. H. Jones vs W. Kendall et al., for assault and battery, for the Quarter session, 183609-15; W. Kendall vs H. Jones, for assault and battery, for the Quarter session, 1836-09-15.
} 
des habitants préfèrent déposer des accusations devant un juge de paix de la ville plutôt que devant un de ceux qui résident plus près d'eux. En fait, prétendait-il, puisque les Canadiens français ignorent le droit anglais, lorsque l'un d'eux apprend qu'un mandat a été émis contre lui à Montréal, il se croit déjà condamné et s'empresse d'offrir une réparation au plaignant ${ }^{35}$. En d'autres mots, le recours en justice n'amène pas nécessairement un dénouement judiciaire, le plaignant pouvant bien utiliser la justice pénale à ses propres fins. L'écart entre le nombre de dépositions et celui des dossiers qui aboutissent finalement devant la cour laisse croire qu'il est toléré que ce recours puisse faire partie de la stratégie du plaignant où un accommodement quelconque peut se substituer à la punition. C'est de cette manière que se règle une autre affaire d'assaut, lorsqu'un aubergiste dépose contre quatre personnes venues dans son auberge pour en battre une autre. Une seule de ces quatre personnes est mise en accusation, mais elle ne se présente pas à la cour. Le document de la déposition est accompagné de la note suivante, signée de la main de l'inculpé: "gentlemen, please excuse my liberty of writing to you a few lines, letting you know that I am lying ill and unable to attend at court. The case against me is settled ${ }^{36}$.»

Il en va de même des dossiers qui entrent plus profondément dans le processus judiciaire. Lors de l'ouverture de la session, les dossiers sont étudiés par le grand jury qui évalue la pertinence des accusations et décide si la preuve de la partie plaignante est suffisante pour qu'il y ait procès. À Québec, le grand jury semble plus enclin à acheminer les dossiers au procès qu'à les rejeter. Au dernier jour de la session, la cour détermine les sentences de ceux qui ont été trouvés coupables. Presque chaque session, on trouve une liste de prisonniers qu'il faut relâcher, faute de poursuivant. D'autres dossiers sont rejetés par le grand jury durant les audiences («no bill»), mais un certain nombre d'entre eux le sont aussi au dernier jour de la session. Tout porte à croire que la poursuite tombe parce que la partie plaignante est prise en défaut (défaut de comparaître, refus des témoins de se présenter...) ou refuse tout simplement de donner suite à l'affaire. D'ailleurs, dans leurs représentations, les grands jurys des sessions de la paix déplorent régulièrement l'absence des témoins et plaignants au moment du procès et regrettent qu'on ne prenne pas de mesures pour les forcer à

35. Archives nationales du Canada (à l'avenir ANC), RG4 B14 vol.3 : Captain Wetherall to W. F. Coffin, 1840-06-03.

36. Le mot est signé M. Daly, Woolfs Cove, 1836-10-20. Il se trouve dans le dossier: W. Quinn vs M. Broughton and others, for assault \& c., for the Quarter Sessions, 1836-09-26. Voir aussi le registre. 
comparaître $^{37}$. Même à la cour du banc du Roi, où le procureur et le solliciteur général poursuivent les plus importantes infractions au nom du roi, le grand jury a aussi l'occasion de se plaindre de l'absence des témoins ${ }^{38}$. Dans la plupart des cas, la déposition engage par cautionnement les poursuivants à se présenter à la cour. À cet effet, lors de la session de janvier 1836, la cour devra enjoindre au greffier de prendre les mesures nécessaires afin que les montants des cautionnements qui n'ont pas été respectés soient prélevés, en plus de demander au gouverneur général son avis sur la question. Toutefois, la cour précise que cela ne s'applique pas aux infractions d'assaut et batterie et à toute autre infraction qui n'est pas de nature publique ${ }^{39}$.

Ce scénario est repris de sessions en sessions au cours des années 1830. Peu de dossiers d'assaut parviennent jusqu'au procès ${ }^{40}$. Par exemple, au cours de l'année 1831 (quatre sessions), le registre indique que seulement 77 dossiers d'assaut furent soumis à la cour qui rendra 28 verdicts de culpabilité ; pour toute l'année 1836, 67 dossiers d'assaut aboutiront à 23 verdicts de culpabilité.

Certes, ces dossiers qui tombent avant une mise en accusation formelle, puis les autres en cours de procédure, peuvent ne mener à aucun règlement judiciaire ou infrajudiciaire. Mais quel que soit leur dénouement, il est clair que l'application de la loi en matière d'assaut repose davantage sur la stratégie des plaignants que sur l'adhésion à une logique judiciaire répressive et serrée. La justice pénale est pleine de retenue devant ces affaires qu'elle qualifie de «privées». Les plaignants, en mettant fin à la poursuite, partagent un certain pouvoir sur la manière dont s'administre la justice pénale et par voie de conséquence, sur ce que constitue un assaut du point de vue légal ${ }^{41}$.

37. Voir les représentations du grand jury des sessions de la paix (à l'avenir: jury). On trouve certaines de celles-ci éparpillées ou plus rarement transcrites dans le registre (ANQQ, TP12, S1). On en trouve aussi dans la presse et nous y référerons en indiquant le journal en question. Voir Jury, janvier 1836, octobre 1836, avril 1837, mars 1839.

38. Quebec Mercury (à l'avenir $Q M$ ), 1831-10-01. Notons que les plaignants sont appelés «témoins » dans leur propre dossier, puisque la poursuite s'effectue au nom du roi. Sur l'absence des plaignants et témoins, voir A. Steinberg, loc. cit., 72.

39. Voir le registre et la loi de judicature de 1793 (34 Geo.III, c.6).

40. Par un autre mécanisme qui permet de remettre le procès à la session suivante, d'autres dossiers tombent et ne réapparaissent plus dans le registre, mais cette fois c'est le plus souvent la partie inculpée qui prend l'initiative. On appelle cette motion traverse en anglais. Au cours de la session de juillet 1836, par exemple, dix dossiers furent remis à la session d'octobre. Or, pour octobre, le registre ne conserve aucune trace de six d'entre eux. En 1839, on débattra du poids de cette motion en droit. Voir l'opinion des juges dans le registre, 1839-04-30. Voir aussi la correspondance entre le greffier de la paix à Montréal et celui de Québec: Delisle à Scott, 1839-11-20.

41. Cet élagage n'est pas propre à l'assaut. Bien que les poursuites avortent plus fréquemment dans les cas d'assaut, on trouve aussi des cas en matière de larcin. Par exemple, le 30 avril 
Il ne faut pas écarter par ailleurs certains obstacles à ce qu'une poursuite aboutisse devant la cour. En optant pour cette avenue, celle de la mise en accusation, celui qui mène un dossier d'assaut devra débourser les frais de justice; des frais qui s'additionnent pour chacune des démarches qu'implique la poursuite puisque policiers et greffiers retirent des honoraires pour les devoirs qu'ils accomplissent. Le particulier devra donc avancer des sommes considérables qui comprennent au minimum deux chelins et six deniers pour la déposition, cinq chelins pour la sommation, dix chelins pour que le greffier prépare l'acte de mise en accusation (bill of indictment), dix chelins pour le travail du greffier à la cour et ainsi de suite. Comme une telle démarche peut aussi entraîner d'autres honoraires pour l'émission d'un mandat d'arrestation et pour sa mise à exécution, une poursuite peut parfois coûter jusqu'à deux ou trois livres ${ }^{42}$. En revanche, si le plaignant cherche à obtenir un cautionnement pour la paix, il n'aura à débourser que deux chelins et six deniers. Il est donc possible que les frais de justice rebutent bon nombre de plaignants ne pouvant mobiliser les sommes nécessaires pour faire cheminer leur dossier plus avant dans la procédure.

On ne peut cependant s'avancer que prudemment sur cette question des frais. À la session de janvier 1836, on prétend que certains officiers de justice menacent un inculpé de le garder en prison s'il refuse de payer leurs frais pour l'avoir mis sous arrestation, c'est-àdire pour avoir exécuté un mandat. En d'autres mots, pour s'assurer de recevoir leurs honoraires, des officiers de justice menacent l'inculpé d'emprisonnement. À cette occasion, les commentaires de la cour portent spécifiquement sur les infractions qui ne sont pas de nature publique, notamment sur les assauts et batterie. La cour enjoint alors au greffier de ne préparer un mandat d'arrestation à la suite d'une plainte que dans les seuls cas où la partie plaignante avancera les frais associés à l'exécution du mandat $\left(5\right.$ chelins $\left.^{43}\right)$. Si les officiers de

1845, Baptiste Ringuette demande à la cour s'il sera bientôt libéré de la prison où il pâtit depuis le 24 février, car il n'a pas été jugé lors de la session d'avril. Il dit comprendre que le plaignant n'a pas l'intention de se présenter en cour pour le poursuivre. L'accusation concerne le vol d'une montre d'argent. Voir Petition, Baptiste Ringuette, 1845-04-30; Calendar of untried prisoners confined in the common gaol, 1845-04-19.

42. Voir la table des honoraires adoptée par la cour des sessions trimestrielles de la paix en 1830: Quebec Tariff, General Quarter Session of the Peace, 1830-01-19.

43. Quelques années plus tard, ce seront les greffiers qui seront rabroués, justement parce qu'ils se montreront réticents à prendre des dépositions sans que le poursuivant en ait avancé les frais. Cela est significatif de l'application d'une logique différente qui cherche à faciliter la judiciarisation des dossiers. Voir Report of the Special Committee Respecting Fees, 1840-04-01. Voir aussi, lorsque l'assemblée cherche à embarrasser le gouvernement, l'enquête sur le greffier 
justice éprouvent de la difficulté à récupérer leurs honoraires, cela suppose que les frais de justice ne constituent pas toujours une condition nécessaire et préalable à une poursuite.

Enfin, la difficulté pour les plaignants de réunir une preuve suffisante, le fait que certains doivent quitter la ville au moment de la session, que des témoins refusent d'appuyer la poursuite, peuvent aussi expliquer cet élagage considérable des dossiers ${ }^{44}$.

Qu'en est-il de ces assauts qui se soldent par un verdict de culpabilité? D'emblée, ils sont si peu nombreux qu'il y a lieu de se demander si la punition se situe nécessairement dans la sentence de la cour. À bien des égards, le processus judiciaire permet de punir un accusé en souillant sa réputation et ce, même si le dossier ne débouche pas sur un verdict de culpabilité. Ce qui peut être un pis-aller pour celui qui n'a pas réussi à forcer l'autre à négocier une réparation ou encore la satisfaction d'un désir de vengeance. Au lendemain de la Conquête, dans la foulée des débats sur les droits pénal et civil qu'on allait adopter dans la colonie, le procureur général Maseres et Chartier de Lotbinière s'inquiétaient de la facilité avec laquelle, en droit pénal anglais, un particulier pouvait introduire une poursuite en justice contre un homme de condition ${ }^{45}$. Mais peu importe le statut de celui qui fait l'objet d'une poursuite, on reconnaît à l'époque la facilité avec laquelle une accusation sans fondement peut faire son chemin dans le processus judiciaire et on craint que des particuliers utilisent la justice pénale pour satisfaire leurs propres intérêts. C'est d'ailleurs un des arguments qu'évoque Robert Peel en faveur de l'établissement d'un ministère public se chargeant des poursuites criminelles ${ }^{46}$. Il n'est

\footnotetext{
de la paix de Trois-Rivières, David Chisholme, que l'on soupçonne de transformer des dossiers d'assaut et batterie en dossiers d'assaut avec intention de meurtre. Ce qui expliquerait cette «manipulation» du greffier, c'est que l'assaut avec intention de meurtre est considéré comme une affaire publique, le greffier pouvant alors réclamer ses honoraires au gouvernement plutôt qu'au particulier, lequel ne les lui remet pas toujours. Dans, Reports, Correspondence Returns and Other Papers Relating to Canada, Item 270, «Reports and Correspondence on the Subject of the Charges Preferred Against Mr. Chisholme and Judge Fletcher» (Shannon, Irish University Press, Series of British Parliamentary Papers, 1837), 8.

44. Une analyse qui tiendrait compte de certains attributs des plaignants et des accusés, tels leur statut, leur sexe, leur parenté, leur qualité de résidant ou d'immigrant récent, nous permettrait probablement de relever d'autres aspects de cet élagage.

45. Sur leurs opinions, voir Douglas Hay, «Prosecution and Power: Malicious Prosecution in the English Courts, 1750-1850», dans D. Hay and F. Snider, dir. op. cit., 343395; André Morel, «La réception du droit criminel anglais au Québec (1760-1892)», Revue juridique Thémis, 13,2-3 (1978): 497-499.

46. Voir Clive Emsley, Crime and Society in England 1750-1900 (London, Longman, 1987), 148.
} 
donc pas surprenant que certaines dispositions législatives visent spécifiquement à contrecarrer les poursuites malicieuses, notamment à propos des actions pénales où le plaignant pouvait se voir remettre une partie de l'amende. L'argument est aussi soulevé en ce qui concerne les limites d'action, c'est-à-dire le temps dont dispose le plaignant pour déposer sa plainte à partir du moment de l'infraction. On redoute que, pour assurer sa défense, mais aussi pour se placer en position de force, l'inculpé ne réagisse en déposant lui aussi une plainte contre son adversaire, plainte qui pouvait éventuellement concerner une affaire déjà ancienne ${ }^{47}$. Dans son traité sur le droit pénal, Edward Carter ne cesse de mettre en garde les juges de paix contre des démarches de la sorte ${ }^{48}$. En octobre 1841, à propos d'un assaut, Joseph Keth, journalier, dépose "pour la paix » contre un charretier du nom de Voyer. Le juge de paix Symes rejette l'affaire, qu'il justifie de la manière suivante: «found the charge to be frivolous on the part of a young man against an old one, has taken upon honour to discharge the defendant ${ }^{49}$.» L'aspect infamant peut se produire dès la déposition, mais il se trouve aussi activé à divers moments du processus judiciaire $^{50}$. C'est le cas notamment lorsque des inculpés sont relâchés, faute de poursuivant, mais seulement après que l'accusation eut été lue devant la cour.

Enfin, il existe une autre manière par laquelle un accusé peut subir une punition sans pour autant être condamné par la cour. En 1836, William Bradford dépose contre James White devant le greffier de la paix pour un assaut et batterie dont il se dit victime. La déposition indique que l'affaire se destine aux sessions trimestrielles de la paix. Dans ce cas, en théorie du moins, c'est Bradford qui devra payer tous les frais de justice afférents au traitement de sa plainte par les officiers de justice. De plus, ce qui est toutefois exceptionnel dans un tel cas, White est emprisonné depuis le moment de la déposition, le 4 octobre, sous les ordres du juge de paix Robert Symes. Or, dans cette affaire,

47. Pour les actions pénales, voir 52 Geo.III (1812), c.7. À propos des auberges non licenciées, voir 35 Geo.III (1795), c.8, s.20. En droit civil, voir la loi sur les poursuites triviales et vexatoires, 7 Geo.IV (1827), c.6, qui limite le montant des dépens qui peuvent être accordés au demandeur lorsque les dommages ne dépassent pas 40 chelins. À propos de cette dernière, voir les commentaires de William Blackstone, Commentaries on the Laws of England, vol.3 (London, A. Strahan, 1765-69, 1809), 400-401.

48. Edward Carter, A Treatise on the Law and Practice on Summary Convictions and Orders by Justices of the Peace, in Upper and Lower Canada (Montréal, John Lovell, 1856), 56-57 par exemple.

49. J. Keth vs Voyer, for assault, for the peace, 1841-10-19.

50. À propos de la réputation des accusés, voir la charge au grand jury de la cour du banc du Roi de septembre 1810, dans La Gazette de Québec (à l'avenir GQ), 1810-10-04. 
il ne semble pas y avoir de mise en accusation formelle. À la toute fin de la session d'octobre, le 29, James White est tout simplement relâché, faute de "poursuivant», indique la note du greffier dans le registre. Aucun procès n'est intenté contre White, bien qu'il ait été emprisonné pendant près d'un mois. Lors de cette même session, Louise Suzanne Jacques est relâchée faute de poursuivant. Elle avait été emprisonnée le 10 août, sous les ordres du juge de paix Louis Massue, à la suite de la déposition de Michel Tsioui qui disait avoir été l'objet d'un assaut et de menaces ${ }^{51}$.

L'observation du cheminement des dossiers permet de relever tout un mode de justice qui circule ni tout à fait dans un circuit judiciaire clos, ni tout à fait en dehors. Ainsi, en matière d'assauts, la justice pénale telle qu'elle est appliquée au cours des années 1830 fait place aux intentions et stratégies déployées par les particuliers. Il est mal vu de se porter «poursuivant» ou informateur puisque l'opprobre s'attache aussi à celui qui poursuit en justice. Celui-ci peut préférer dans bien des cas laisser planer la menace d'une poursuite en règle afin de favoriser un accommodement hors cour, une entente qui lui rapporte quelque chose. En livrant entièrement son affaire à la justice, le plaignant n'en retire rien, sinon une quelconque satisfaction de remplir son rôle de citoyen, comme certains réformateurs le souhaitent.

Il semble donc que l'on doive considérer les rapports entre le judiciaire et l'infrajudiciaire comme ne formant pas deux ensembles séparés l'un de l'autre. C'est que la justice pénale d'Ancien Régime fonctionne autrement que celle du $\mathrm{XIX}^{\mathrm{e}}$ siècle ${ }^{52}$. Les dossiers d'assaut circulent dans une administration judiciaire flexible où des juges de paix disposent d'un large pouvoir discrétionnaire, où des punitions peuvent être distribuées avant même qu'il y ait un verdict de culpabilité. Administrer la justice en matière d'assaut ne se résume pas à distribuer des verdicts, c'est-à-dire à trancher s'il y a eu ou non crime. La justice pénale ne s'appuie pas sur le principe que toutes les infractions doivent nécessairement mener à un affrontement entre l'État et le citoyen, une idée relativement nouvelle à l'époque ${ }^{53}$. Cette justice pénale est ouverte à ce que des particuliers soumettent leurs litiges à une juridiction criminelle, à ce qu'ils engagent des poursuites, mais elle est réticente à exercer une répression soutenue de tout ce qui vient

51. En fait, sept personnes (six dossiers d'accusation) furent ainsi emprisonnées durant la période qui précède la session d'octobre, puis relâchées sans être poursuivies.

52. Par exemple, voir Douglas Hay, «Property, Authority and the Criminal Law», dans Douglas Hay et al., Albion's Fatal Tree (New York, Pantheon Books, 1975), 17-63.

53. D. Philips, loc. cit., 158. 
à sa connaissance. Le juge de paix W. Hargrave du comté d'Inverness, dans le district de Québec, affirmait: "I have often found a little cautionery advice serve the purpose much better than a prosecution $^{54}$.»

En mettant en place une justice sommaire d'abord dans les cas d'assaut commun, puis dans les cas de petits vols et de quelques autres infractions, l'adoption des lois de Peel en 1841 va frapper de plein fouet ce mode d'administration de la justice. Une procédure plus accessible, plus expéditive et resserrée constituera une nouvelle avenue judiciaire pour les cas d'assaut.

\section{LA RÉFORME DU DROIT PÉNAL}

Les lois de Peel manifestent une volonté de rompre avec un droit qualifié d'ésotérique ${ }^{55}$. Parmi d'autres, Jeremy Bentham souhaite que la loi guide les conduites et, pour cela, il lui faut être claire et connue de tous. C'est une conception positiviste du droit qui inspire les réformes. Selon cette doctrine positiviste, le droit et la justice se résument à la loi et aux tribunaux. Les réformateurs souhaitent rompre avec la flexibilité de la justice, sa tolérance envers divers accommodements, comme les poursuites qui finissent par tomber ou les plaignants et témoins qui refusent de comparaître. Pour William Blackstone, comme nous l'avons vu plus haut, les poursuites pénales doivent avoir pour fins de réaliser la justice publique, non pas les intérêts des particuliers qui les engagent ${ }^{56}$. Ainsi, cette justice publique, dont la loi est l'expression de la volonté générale, doit se présenter comme si elle était détachée des rapports de force ${ }^{57}$. Tout cet infrajudiciaire qui peut passer provisoirement par le judiciaire tend alors à être conçu comme s'il se situait en dehors de la justice.

54. Walter Hargrave to Perrault \& Scott, 1839-12-04. Cette lettre accompagne son rapport trimestriel du 19 octobre 1839 .

55. Dans les mots de Jeremy Bentham, «right is the conformity to a rule, wrong the deviation from it», cité dans Michael Lobban, The Common Law and English Jurisprudence 1760-1850 (Oxford, Clarendon Press, 1991), 121. Voir, pour l'Angleterre, les travaux des différents comités formés pour étudier la loi criminelle, dans Committee on Criminal Law (Shannon, Irish University Press of British Parliamentary Papers, 1968).

56. Voir aussi les arguments de J. Chitty en ce qui concerne l'obligation morale de poursuivre, op. cit., 2-3.

57. Journaux de la chambre d'assemblée de la province du Bas-Canada, Rapport du comité spécial [...sur les...] pénitenciers, App. FFF, 1836. Voir aussi comment on présente la loi lors de l'enquête sur le décès du détenu J. Collins dans la prison de Montréal: «le plus faible comme le plus puissant [...] ont également droit à la protection et à la vigilance de nos Lois.» Voir Journaux de la chambre d'assemblée de la province du Bas-Canada, Rapport du comité spécial [... sur...] la mort du nommé John Collins..., 1835-36. 
L'efficacité de la justice pénale s'envisage maintenant dans la capacité de l'appareil de justice de détecter, d'accueillir, puis de traiter toutes les infractions. Le préambule d'une des lois de 1841, celle qui concerne les félonies, indique précisément qu'il faut améliorer la justice criminelle de manière à assurer la punition des coupables ${ }^{58}$. On insiste pour que la justice s'administre en adoptant pour principes que toutes les infractions fassent l'objet d'une répression et que la réaction contre les infractions soit prompte.

Dans la colonie bas-canadienne, on relève ça et là des traces de ce discours de réforme, plus clairement exprimé et débattu dans la métropole. On trouve ainsi des échos de l'utilitarisme des Bentham et Beccaria $^{59}$ dans certains propos, notamment ceux des grands jurys. Il est aussi question de l'incertitude qui plane sur le processus judiciaire et de l'espoir de l'impunité que l'on décrie de plus en plus fermement comme un défaut du code anglais, compte tenu de peines que l'on dit maintenant trop sévères ${ }^{60}$. On souhaite substituer la certitude de la punition à la sévérité du châtiment ${ }^{61}$.

Certes, le débat s'exprime plutôt à propos des infractions les plus sérieuses. Cependant, ces intentions de rompre avec une administration de la justice perçue comme défectueuse parce que trop flexible accompagnent un projet de réforme des masses qui s'élabore, depuis un bon moment, en un discours sur le désordre urbain, sur la prolifération du vice et sur les épidémies. À cet égard, des médecins s'immiscent dans les questions concernant l'ordre moral et social ${ }^{62}$. Ces pressions tentent notamment de légitimer le recours croissant à l'emprisonnement contre tous ceux qui «troublent la paix ${ }^{63} »$.

58. Dans le préambule d'une des grandes lois de réforme adoptées en 1841,4 \& 5 Vict.(1841), c.24.

59. Rappelons les mots de Beccaria: «Un des moyens les plus sûrs de réprimer les délits, ce n'est pas la rigueur des châtiments, mais leur caractère infaillible [...]. La certitude d'une punition, même modérée, fera toujours plus d'impression que la crainte d'une peine terrible si à cette crainte se mêle l'espoir de l'impunité.» Dans Cesare Beccaria, Des délits et des peines (Genève, Librairie Droz, 1764, 1965), 46. Voir aussi W. Blackstone, op. cit., 4: 16.

60. Voir ces représentations des grands jurys: $Q M$ 1826-09-19; GQ 1821-10-01. Dans cette dernière, le grand jury prétend que de recourir trop facilement à l'emprisonnement sans preuves suffisantes contribue à accroître l'incertitude. D'autres exemples dans le roman de François-Réal Angers, Les révélations du crime (Réédition-Québec 1834, 1969), 10-11 et dans Journaux de la chambre d'assemblée de la province du Bas-Canada, Rapport du comité spécial [...sur les...] pénitenciers, App. FFF, 1836.

61. Par exemple, la représentation du grand jury de la cour du banc du Roi de Montréal, dans $Q M$ 1826-09-26.

62. André Cellard, Histoire de la folie au Québec, de 1600 à 1850 (Montréal, Boréal, 1991).

63. Voir M. Dufresne, op. cit. et J.-M. Fecteau, op. cit., 173-206. 
Au cours des années 1830, les grands jurys insistent pour que les querelles entre particuliers soient réglées promptement et autrement qu'en mobilisant la cour et ils vont même jusqu'à réclamer un magistrat de police. Ils se plaignent aussi de plus en plus de l'absence des témoins, disions-nous. Cela nous semble le signe de l'appui que les jurys vont donner à l'introduction des lois de Peel ${ }^{64}$.

Le resserrement de l'administration de la justice pénale va s'effectuer de deux manières. D'une part, les juges de paix de la ville seront progressivement mis à l'écart de l'administration de la justice. La cour des sessions trimestrielles de la paix perdra de son importance alors qu'une proportion importante des affaires seront traitées à la cour de police. Ce réaménagement juridictionnel va s'opérer en plaçant à nouveau un magistrat de police dans la ville. D'autre part, du point de vue de la procédure et des avenues judiciaires, la loi va maintenant permettre de disposer rapidement et à moindre frais des dossiers d'assaut. Ce même magistrat de police va s'approprier ces dossiers. Nous le verrons en commençant par les juridictions.

Dès 1810 , sous le «règne de terreur de Craig ${ }^{65}{ }^{\text {» }}$, le gouvernement central s'était efforcé de s'immiscer dans la justice locale, celle des juges de paix, en nommant Ross Cuthbert président des sessions de la paix. Celui-ci cherchera à pousser magistrats et jurés à appliquer la loi plus fermement en leur rappelant leurs devoirs ${ }^{66}$. Soudainement, les journaux vont porter une attention plus soutenue à la justice pénale, attention que l'on expliquerait à tort comme une réaction directe à une augmentation du crime. Au même moment, en cour des sessions de la paix, on va se mettre à faire porter les frais de justice par le coupable dans les cas d'assaut. Cette mesure vise à encourager les plaignants à traduire en justice les contrevenants puisqu'elle permet de poursuivre à moindre coût ${ }^{67}$. Il suffit de quelques années pour qu'on abandonne cette pratique ${ }^{68}$. Des critiques à l'endroit des juges de paix se font entendre à nouveau ${ }^{69}$; puis, les journaux accordent moins d'attention

64. Jury, octobre 1838 ; juillet 1838 ; avril 1841 ; octobre 1841 ; janvier 1845.

65. F. Murray Greenwood, Legacies of Fear (Toronto, University of Toronto Press, 1993), 228-246.

66. $G Q 1810-05-17, G Q 1810-07-05$. Voir aussi les nouveaux règlements de police, $G Q$ 1810-02-01.

67. Ce n'est pas le cas lors de la session d'avril 1810, mais c'est le cas pour les sessions suivantes. Voir GQ 1810-05-03; 1810-07-26; 1810-11-01; 1811-05-02; 1812-01-23.

68. Il n'en est plus question à la session de janvier 1816, GQ 1816-01-25. Le registre de la cour, qui est une source plus fiable, n'indique pas qu'on impose des frais au cours des années 1830.

69. En 1815, la Gazette de Québec rapporte que la session qui devait s'ouvrir le 21 octobre ne l'est toujours pas le 26 du même mois «faute d'un Quorum de Juges de Paix». GQ 1815-10-26. 
aux sessions de la paix. Au début des années 1830, le poste de président des sessions de la paix est aboli, de fait, puisque l'Assemblée refuse de voter le salaire de son titulaire, Robert Christie ${ }^{70}$.

Il faut attendre les rébellions pour que le gouvernement central place à nouveau un magistrat stipendiaire au centre de l'administration de la justice pénale urbaine. L'ordonnance de police de Durham rétablit ce poste qui sera confié à Thomas Ainslie Young ${ }^{71}$. Celui-ci ne pourra siéger à la cour des sessions trimestrielles de la paix comme c'était le cas auparavant, mais la «nouvelle» force de police est placée sous son autorité. À partir de la cour de police (bureau de la paix), ce magistrat va rapidement s'imposer comme le gardien de la porte d'entrée de la justice pénale, doublant ainsi les juges de paix au moment où s'enclenche une poursuite. D'ailleurs, il rapporte régulièrement au commissaire de police du Bas-Canada le nombre des dossiers qu'il parvient à acheminer au procès et ceux qui se soldent par une condamnation. Lorsqu'en 1838 les greffiers de la paix auront à justifier l'augmentation des honoraires réclamée au gouvernement, ils expliqueront que tous les dossiers font maintenant l'objet d'une procédure formelle. Ainsi, suggèrent-ils, ce qui peut paraître comme une augmentation du crime, s'explique par une répression plus soutenue de la part de la nouvelle police et du surintendant, puis par un traitement plus serré des plaintes ${ }^{72}$.

Les juges de paix subissent des pressions afin qu'ils agissent de manière plus formelle. On dénonce leur ignorance du droit et leurs absences en cour, notamment en cour des sessions hebdomadaires de la paix $^{73}$. Les dossiers doivent être repoussés de semaine en semaine, dit-on, compte tenu de l'absence d'un des juges. L'ordonnance du conseil spécial qui institue la police rurale accordera des pouvoirs extraordinaires aux magistrats de police, leur permettant d'exercer,

70. Voir l'enquête au sujet de Robert Christie dans Journaux du conseil législatif du BasCanada, 1829, App. D.D. Les témoins entendus lors de l'enquête suggèrent qu'en matière de droit pénal, Christie fait fi de l'opinion des juges de paix, ce qui irrite ceux-ci au point où ils délaissent leurs devoirs. Cette affaire qui est aussi relative à la liste des juges de paix qui devront composer la nouvelle commission vaudra à Christie les foudres de la Chambre d'assemblée, dont il sera expulsé à plusieurs reprises. Voir aussi Robert Christie, A History of the Late Province of Lower Canada (Montréal, R. Worthington, 1866), III : 241-251.

71. 2 Vict.(1838), c.2.

72. Draught of our answers to the remarks on the a/c for the half year ending 10 october 1838, Quebec, 1838-11-26, Perrault \& Scott.

73. Jury, octobre 1838. Chaque semaine, deux juges de paix doivent siéger en cour des sessions hebdomadaires de la paix afin d'entendre des poursuites relatives à la police urbaine. C'est là que des constables poursuivent ceux qui vendent de l'alcool sans licence, que l'inspecteur des chemins poursuit ceux qui négligent d'enlever la neige devant leur habitation, et autres affaires du genre. Voir le statut 34 Geo.III, c.6. 
dans certains cas, les pouvoirs de plus d'un juge de paix. Cela signifie que T. A. Young pourra en fait siéger seul en cour des sessions hebdomadaires de la paix où, rappelons-le, on juge les affaires de police urbaine. Le magistrat se voit conférer ce pouvoir par 4 Vict.(1840), c.47, pouvoir qu'il perd en 1842 au moment de l'abolition de la police rurale (6 Vict., c.14), mais qu'il recouvre en 1851 (14 \& 15 Vict., c.95, s. $\left.29^{74}\right)$. Lorsqu'ils en auront l'occasion, les juges de paix affirmeront remplir leur devoir avec zèle et insisteront sur l'aspect volontaire de leur contribution à la justice urbaine, c'est-à-dire sans la rémunération consentie à l'inspecteur et surintendant ${ }^{75}$. Quant à l'obligation de rapporter trimestriellement toutes leurs activités judiciaires, ce qu'imposait une ordonnance du conseil spécial de 1839, les greffiers remarqueront que la grande majorité des juges refusent de s'y conformer ${ }^{76}$.

Le juge de paix Robert Symes est l'un de ceux qui rivalise avec l'inspecteur et surintendant de police et la police qu'il dirige. Au début de l'été 1840, il lance une poursuite contre le chef de police Robert Henry Russell, l'accusant d'avoir refusé d'exécuter un mandat ${ }^{77}$. Le commissaire de police, W. Coffin, expliquera à R. H. Russell qu'il fallait prévoir des tensions de la sorte avec les juges de paix et qu'il devra garder un œil sur R. Symes ${ }^{78}$. À un autre moment, en l'absence de T. A. Young à la cour de police, R. Symes remet en liberté sept «femmes de mauvaise vie» que Young avait fait arrêter. Offensé par cette manœuvre, ce dernier envoie à nouveau des policiers cueillir ces femmes près de la caserne logée dans l'ancien collège des jésuites de la haute-ville. Il rapporte au commissaire de police avoir coffré onze d'entre elles pour une période de deux mois en vertu de l'ordonnance de police ${ }^{79}$. Une altercation semblable oppose le juge de paix Bigaouette et le chef de police Russell, lorsque Bigaouette interdit à

74. Pour le Haut-Canada, voir J. C. Weaver, op. cit., 68-69. Au cours des années 1850, le greffier sera lui aussi rémunéré sur le mode du salaire plutôt que selon des honoraires. Voir 13 \& 14 Vict. (1850), c.37, puis 16 Vict. (1853), c.196. Comme le dit J. C. Weaver, on remplace les amateurs par des professionnels.

75. D'autres exemples de plaintes dans: Clerks of the peace to civil secretary, 1839-0225; W. Bennett, city treasurer, to G. O. Stuart, Mayor of Quebec, 1848-08-02. La réplique des juges de paix se trouve dans Report, Magistrates' room, 1848-09-14.

76. 2 Vict., c.20 amende 4 Geo.IV (1824), c.19, selon laquelle les juges de paix devaient rapporter les amendes qu'ils imposent. ANC RG4 B18 vol.1 : Perrault \& Scott, 1841-07-22. En 1852 , le secrétaire fait parvenir un formulaire et une copie de l'ordonnance de 1838 aux juges de paix qui ont manqué à ce devoir. Voir ANC RG4 B18 vol.1.

77. Voir l'acte d'accusation: Regina vs Robert Henry Russell, July session 1840.

78. ANC, RG4 B14 vol.29, Private instructions to inspector Russell, 1840-08-18.

79. Young to Coffin, 1839-01-30. 
un policier de se saisir d'une voiture laissée sans surveillance. Cette fois, le commissaire Coffin suggère à Russell de poursuivre le juge de paix, afin de lui donner une bonne leçon, dit-il, mais seulement s'il est sûr d'obtenir un verdict de culpabilité ${ }^{80}$.

Ces frictions témoignent du bras de fer que se livrent des juges de paix et le gouvernement central par le truchement de la police, de son chef, puis de l'inspecteur et surintendant de police. Celui-ci aura éventuellement raison des juges de paix, et occupera une place de plus en plus importante dans l'administration de la justice pénale urbaine. Ainsi, lorsqu'il perd sa double juridiction en 1842 (mais qu'il recouvre ses pouvoirs sur la police), l'inspecteur occupe un des deux sièges de la cour des sessions hebdomadaires de la paix ${ }^{81}$. À partir de 1856 , une nouvelle juridiction, celle du recorder, va aussi empiéter sur la place des juges de paix, en se substituant à la cour des sessions hebdomadaires de la paix ${ }^{82}$.

En parallèle à cette liquidation formelle de la justice pénale d'Ancien Régime, l'adoption des lois des Peel va faire converger les dossiers de justice pénale vers la cour de police, notamment les dossiers d'assaut. Ceux-ci pourront y être jugés sommairement parce que, avec l'accord du plaignant, la loi permet à un juge de paix seul de convoquer les parties et de rendre un jugement ${ }^{83}$. En pratique, dans la ville de Québec, comme l'inspecteur et surintendant de police siège en permanence à la cour de police, c'est lui qui préside lors de ces procès. En accordant à un juge de paix seul le pouvoir d'entendre des méfaits de la sorte, on vise à accélérer le dénouement des poursuites. Dans les affaires d'assaut, l'inspecteur et surintendant de police, qui dirige aussi le corps de police, peut alors jouer le rôle de juge d'instruction et de juge de jugement. Au détriment des garanties juridiques dont les jurys devaient être les dépositaires, on s'assure d'une justice expéditive et moins incertaine. Bientôt aussi, d'autres infractions sont dirigées vers une procédure sommaire. C'est le cas des petits larcins en

80. ANC RG4 B14 vol.29, Police Instructions: 1841-03-19, 1841-04-30.

81. Voir trois lettres reproduites dans celle du maire de Québec, G. O. Stuart, dans G. O. Stuart to R. B. Sullivan, secretary, 1848-08-11; puis la réplique des juges de paix dans Report of the Committee, 1848-09-14.

82. 19 \& 20 Vict., c.106. La cour du recorder se substitue à la cour des sessions hebdomadaires de la paix. La cour peut aussi entendre les affaires d'assaut et exécuter l'ordonnance de police.

83. C'est aussi le cas pour quelques autres infractions: vol de haie, de clôture, de végétaux dans les jardins, etc. En Angleterre, deux juges de paix pouvaient entendre ces affaires, alors qu'au Bas-Canada, un juge seul avait le même pouvoir. 
1857 (20 Vict., c.27), puis de certains «assauts graves » et «tenir une maison de désordre» en 1858 (22 Vict., c.27) ${ }^{84}$.

Le choix de cette avenue sommaire s'accompagnait néanmoins d'un droit d'appel à la cour des sessions trimestrielles de la paix, lequel devait contrebalancer l'absence du jury. Toutefois, il est intéressant de constater que l'acte de judicature de 1857 (20 Vict., c.44) permet soit au surintendant, soit au recorder, de présider seul la cour des sessions trimestrielles de la paix. Le registre montre que ces deux personnages vont siéger alternativement pour entendre les appels en provenance des cours du recorder et de police. Ainsi, l'un entend les appels des procès que l'autre a présidé. On est alors bien loin de la procédure suivie avant la réforme.

Par ailleurs, cette législation formalise l'octroi des dépens à l'une des parties, mesure temporairement appliquée à partir de 1810. En permettant au juge d'impartir les frais de justice, les affaires d'assaut conservent un statut privé, mais à l'intérieur d'un cadre pénal. En d'autres mots, le plaignant court la chance que ses frais de justice les frais d'avocat pour l'essentiel — soient payés par l'accusé, ce qui allège le fardeau du plaignant. Par contre, si le juge de paix considère une poursuite comme étant malicieuse, il peut tenir le plaignant responsable des frais.

S'ajoute aussi, en 1846, une nouvelle loi permettant de forcer les témoins à comparaître (9 Vict., c.5). Il semble qu'avant cette date, en ce qui concerne les méfaits («misdemeanour»), un juge ne dispose d'aucun recours pour forcer un témoin (autre que le plaignant) à comparaître s'il n'y a pas de texte de loi particulier qui accorde ce droit $^{85}$. Les juges ont dorénavant le pouvoir d'emprisonner pendant 10 jours ceux qui refusent de témoigner. Ainsi, en septembre 1846, le nouvel inspecteur et surintendant William K. McCord peut-il émettre un mandat d'arrestation contre A. Chamberland et un certain Caron, témoins qu'il avait sommés de comparaître dans la cause de G. Caron contre T. Dunford pour assaut et batterie, mais qui ne s'étaient pas présentés ${ }^{86}$.

Ces dispositions qui permettent d'accélérer le processus judiciaire et qui concentrent une bonne part de l'administration de la justice dans les mains de l'inspecteur et surintendant de police, favorisent un

84. La procédure sommaire est uniformisée dès 1851 par l'adoption des «Jervis Acts» 14 \& 15 Vict., c. 95, c. 96.

85. C'est l'opinion de E. Carter, op. cit., 157-158.

86. Warrant to apprehend Alexandre Chamberland and one Caron, for neglecting to appear as witnesses, septembre 1846. 
dénouement pénal dans les affaires d'assaut et vont à l'encontre des pratiques de réparation et de punition sans verdict que tolérait auparavant le processus judiciaire. Dès la mise en vigueur de la réforme en 1842 , le nombre des affaires d'assaut qui aboutissait devant les sessions trimestrielles de la paix diminuera rapidement. Pour toute l'année 1841, donc l'année qui précède la réforme, on trouve 64 dossiers d'offenses contre la personne en cours des sessions de la paix. Dix années plus tard, en 1851, il n'y a plus que 15 dossiers. C'est l'inspecteur et surintendant de police qui se met immédiatement à disposer sommairement des affaires d'assaut. Les rapports annuels d'infractions rédigés par le chef de police indiquent un volume d'environ 350 procès sommaires par année durant les années 1840 . Le rapport de l'année 1845 révèle que 491 dossiers d'assaut furent amenés devant l'inspecteur et surintendant de police ${ }^{87}$. On sait aussi que, durant la même année, il a imposé 222 cautionnements pour la paix et tenu 353 procès sommaires. On ne sait toutefois pas précisément combien de ces dossiers concernent spécifiquement des assauts, mais un survol dans les archives nous invite à croire qu'il s'agit de la majorité des cas. Ainsi, non seulement le nombre de causes d'assaut qui aboutissent à un verdict de la cour augmente-t-il considérablement, mais on peut aussi croire qu'un certain nombre de causes, qui auparavant auraient été réglées par un cautionnement pour la paix, se terminent maintenant par un verdict ${ }^{88}$.

La réforme entraîne diverses conséquences. Elle parvient d'abord à transférer les affaires d'assaut vers une juridiction expéditive, celle de l'inspecteur et surintendant de police, mettant ainsi au rancart une large partie du rôle des juges de paix en matière pénale. En distribuant les dépens, elle invite aussi les particuliers à poursuivre puisque leur affaire peut maintenant être traitée à peu de frais et plus rapidement. En ce sens, la réforme a pour effet de criminaliser davantage l'assaut.

Le resserrement du processus judiciaire subjugue davantage celui qui dépose une plainte dans l'engrenage de la justice. Toutefois, il faut être prudent, et ne pas imaginer que l'inspecteur et surintendant de police se met à condamner tout ce qui aboutit devant lui. Il lui arrive

87. On trouve des quantités semblables de dossiers au cours des années suivantes. Voir Archives de la ville de Québec, Police administration, VM 7-1-3-1, Statistics of Crime and Offences in the City of Quebec, R. H. Russell.

88. Il est intéressant de noter qu'au cours des années 1850 , on précise dans les dépositions visant un cautionnement pour la paix que le plaignant ne cherche pas à vexer l'autre partie. On indique alors: «ne cherche pas à vexer» ou «without malice», comme c'est le cas dans la poursuite de Elmire Gagne (Bertrand) contre Séraphin Bertrand, For assault, for the peace, 1851-09-03. 
d'acquitter des accusés et d'imposer les frais au plaignant. C'est le cas pour François Nadeau, lorsqu'il intente une poursuite contre Jos Picard à propos d'un assaut et batterie. Nadeau est condamné à une amende de 5 chelins en sus des frais ${ }^{89}$. Lorsque Marie Dionne accuse T. Corrigan d'assaut et batterie, mais qu'elle ne se présente pas au procès, le surintendant lui impose de payer à Corrigan la somme de 12 chelins pour ses frais ${ }^{90}$. Il arrive aussi que le surintendant rejette la plainte et qu'il impose aux deux parties de payer chacune sa part des frais. Enfin, il arrive encore que le surintendant se plie à la décision des parties qui en sont déjà venues à un accord, auquel cas il indique sur le dossier «settled». Si l'on conserve une certaine marge de manœuvre, il n'en demeure pas moins qu'un volume beaucoup plus considérable de dossiers va passer dans les mains de l'inspecteur et surintendant de police et aboutir à un verdict.

Ainsi à la fin des années 1850, à Québec, les causes d'assaut sont acheminées soit devant le recorder, soit devant l'inspecteur et surintendant de police, deux officiers des gouvernements central et municipal. Les greffiers touchent alors des salaires plutôt que des honoraires. Dans ces cours de justice, il suffit de quelques jours pour qu' un de ces officiers rende un verdict de culpabilité ou d'acquittement. À la même époque, le gouvernement légifère sur l'érection de 19 nouveaux districts judiciaires où seront construits des palais de justice et une prison attenante ${ }^{91}$.

Bien entendu, faute de moyens pour découvrir le nombre réel des assauts, catégorie d'infractions qui peut d'ailleurs être comprise de manière fort large, on ne saurait évaluer le succès de cette politique sur le nombre réel des assauts. Par ailleurs, la réforme retire partiellement la justice des mains des particuliers en limitant leur marge de manœuvre. Ceux-ci ont dès lors moins l'occasion de négocier une entente. Ainsi, l'assaut prend-il une place plus grande dans ce que l'on qualifie dorénavant de «criminalité », dans ce que l'on entend par crime du point de vue public: il s'agit un peu moins d'une affaire privée.

La réforme témoigne de l'adoption d'une politique beaucoup plus axée sur la certitude - la certitude d'un verdict de cour davantage que l'obligation d'une punition -, politique qui implique d'établir des conditions propices au dénouement d'une affaire par un procès. Or,

89. F. Nadeau vs Jos Picard, assault and battery, for a summary trial, 1846-09-09.

90. M. Dionne vs T. Corrigan, for assault and battery, 1855-09-12.

91. Jacques Laplante, Prison et ordre social au Québec (Ottawa, Presses de l'Université d'Ottawa, 1989), 126-127. 
pour bon nombre de dossiers, la tendance de cette réforme va rigoureusement vers un dénouement pénal — entendre punitif - plutôt que vers un mode de justice faisant place aux accommodements, lesquels sont favorisés par l'ouverture à la négociation. Les particuliers ne cessent pas pour autant d'intenter des poursuites, mais la justice d'État va plutôt s'exercer par une vengeance sur un mode punitif.

\section{CONCLUSION}

$\mathrm{Au}$ Bas-Canada, au rythme des transformations économiques, politiques et sociales de la première moitié du $\mathrm{XIX}^{\mathrm{e}}$ siècle, souffle un vent de réforme qui mène à certaines transformations de l'administration de la justice pénale. Le changement du régime des peines s'accompagne d'une liquidation précipitée du mode d'administration de la justice d'Ancien Régime. Les avenues judiciaires, au moins en ce qui concerne les affaires mineures et en particulier les assauts communs, se restructurent. Sur le plan des juridictions, on change et les officiers et les tribunaux. C'est toujours le particulier qui a la responsabilité de poursuivre, mais la justice pénale tend à traiter ce type de dossier sommairement. Cela ne veut pas dire que les particuliers cessent pour autant de déposer des plaintes, mais les options diminuent. Cela ne signifie pas non plus que les accommodements disparaissent, ou même qu'on ne poursuit plus pour se venger. Ce qui décline, pourtant, c'est cet infrajudiciaire qui colonisait l'administration de la justice pénale, qui se mettait en œuvre en se servant d'elle. Cela tend à faire passer l'assaut du domaine privé au domaine public.

L'idée de certitude, chère aux réformateurs des années 1820 et 1830 , a sans doute inspiré et favorisé la réforme en matière d'assaut commun, comme elle a participé à la mise à l'écart des juges de paix. Après l'épisode de 1838 à 1842 où elle s'est concrétisée dans toute sa rigueur, elle a surtout permis de resserrer le processus judiciaire en neutralisant certaines ouvertures propices à la négociation. Enfin, cette réforme s'est inscrite dans le mouvement par lequel s'est façonnée une certaine justice publique qui allait se substituer à la justice royale, en se présentant comme l'ossature du bien-être collectif. 